\title{
Teorias evolucionistas da depressão: panorama e perspectivas
}

\author{
Andreza Conceição de Souza Tavares ${ }^{a *}$ (iD \\ Rebeca Fernandes Ferreira Lima ${ }^{b}$ (i) \\ Rosana Suemi Tokumarua
}

aUniversidade Federal do Espírito Santo, Departamento de Psicologia Social e do Desenvolvimento, Vitória, ES, Brasil

'Universidade Salgado de Oliveira, Niterói, RJ, Brasil

\begin{abstract}
Resumo: A depressão tem alcançado níveis epidêmicos ao redor do mundo. Seria a depressão um distúrbio mental, como é consenso na saúde mental? Teóricos evolucionistas têm-se questionado quanto à função da depressão e proposto modelos específicos para explicá-la. O objetivo deste artigo é apresentar teorias evolucionistas da depressão, discutir as relações de complementariedade e contraposição entre elas e as implicações sociais e práticas para o tratamento da depressão. Essas reflexões e questionamentos no domínio da saúde mental podem influenciar novos estudos a partir de uma perspectiva não patológica da depressão. No âmbito da psicologia essa perspectiva fornece subsídios para repensar a psicoterapia com o deprimido, enfocando a análise causal e a resolução de problemas. Sugere-se a realização de estudos empíricos com a finalidade de testar e sistematizar as teorias evolucionistas da depressão.
\end{abstract}

Palavras-chaves: psicologia evolucionista, depressão, adaptação, desregulação, saúde mental.

A depressão é uma alteração afetiva caracterizada pela presença de humor deprimido (disfórico) e anedonia (capacidade reduzida de ter prazer) (American Psychiatric Association [APA], 2014). Exerce forte impacto sobre a vida social dos indivíduos, tais como a piora nas relações interpessoais e no desenvolvimento de papéis sociais, bem como o declínio das funções neurocognitivas (Sloman, Gilbert, \& Hasey, 2003). A depressão é uma das principais condições emocionais para a qual as pessoas procuram ajuda (Andrews \& Thomson, 2009). A depressão também é o principal fator para mortes por suicídio (World Health Organization [WHO], 2017).

Dados epidemiológicos da Organização Mundial de Saúde (OMS) constatam prevalência da depressão na população mundial em torno de $4,4 \%$, o equivalente a mais de 300 milhões de pessoas, indicando aumento de mais de 18\% entre 2005 e 2015 (WHO, 2017). Os fatores causais da depressão incluem causas genéticas, ambientais e psicológicas, porém o risco de tornar-se deprimido é aumentado pela pobreza, desemprego, eventos de vida, como morte de alguém querido, rompimento de relacionamentos, doença física e uso de álcool e drogas (WHO, 2017). Dessa forma, tendo em vista a alta incidência da depressão ao redor do mundo e suas consequências biopsicossociais, a depressão continua a ser um tema de estudo de grande relevância.

Na psicologia, observou-se o crescente interesse pela compreensão dos mecanismos evolutivos das emoções (Kennair, 2003). Nesse campo, a depressão

*Endereço para correspondência: drezacsandreza@hotmail.com tem recebido uma atenção especial pela psicologia evolucionista (Kennair, 2018), que tem por base a teoria da evolução por seleção natural, proposta inicialmente por Charles Darwin. Essa abordagem sustenta três pressupostos fundamentais para a compreensão do ser humano: (1) existe uma natureza universal com preeminência dos mecanismos psicológicos sobre os comportamentos; (2) os mecanismos psicológicos são adaptações moldadas pela seleção natural; (3) "a estrutura evoluída da mente humana é adequada para o modo de vida de caçadores coletores" (Cosmides, Tooby, \& Barkow, 1992, p. 5).

A psicologia evolucionista interpreta o comportamento manifesto como produto de mecanismos psicológicos selecionados ao longo da evolução humana e, portanto, adaptado ao ambiente de adaptabilidade evolutiva (AAE). O AAE refere-se ao conjunto de condições que estiveram presentes ao longo do período de evolução humana e que atuaram como pressão seletiva (Yamamoto, 2018). Dado que essas condições foram predominantes ao longo da evolução humana e que as condições modernas são muito recentes para atuarem como pressão seletiva, pressupõe-se que o comportamento manifesto nem sempre é adaptativo nas condições modernas (Yamamoto, 2018). Assim, ao tratar de determinada temática a partir da abordagem evolucionista do comportamento humano, busca-se compreender a função adaptativa e o processo de seleção dos mecanismos psicológicos que regulam o desenvolvimento de certas estratégias comportamentais (Izar, 2018). Por função adaptativa entende-se a contribuição do mecanismo psicológico para 
a resolução de problemas de sobrevivência e reprodução dos indivíduos que o exibiram no AAE (Varella \& Valentova, 2018).

A psicologia evolucionista não é a única a estudar o comportamento humano a partir da teoria da evolução proposta por Darwin. Outras abordagens incluem a etologia, a ecologia comportamental humana e a coevolução gene-cultura (Laland \& Brown, 2002). Entretanto, as contribuições da psicologia evolucionista sobre os mecanismos psicológicos selecionados no AAE fornecem a base para elaborar novas perguntas sobre a natureza do transtorno mental e podem contribuir para a redução de epidemias, como a depressão (Abed, Ayton, John-Smith, Swanepoel, \& Tracy, 2019). Tratar e prevenir a depressão requer o reconhecimento das necessidades e vulnerabilidades do ser humano (Gilbert, 2006).

Diversos modelos evolucionistas explicativos vêm sendo apresentados em diferentes revisões (Nettle, 2004). Essas revisões tendem a ser restritas no sentido de que apresentam e discutem os modelos que se referem às propostas teóricas dos autores, pois servem de contraponto ou suporte para a elaboração destas. No Brasil é escassa na literatura a discussão da depressão sob a perspectiva evolucionista (Kennair, 2018; Resende, 2011; Silva, 2008) e não encontramos nenhuma revisão específica sobre o tema nesta abordagem. Dessa forma, dentre os modelos evolucionistas discutiremos nesta revisão: (1) modelos adaptacionistas, para os quais a depressão é adaptativa (Andrews \& Thomson, 2009; Hagen, 1999, 2002, 2003; Price, Sloman, Gardner, Gilbert, \& Rohde, 1994; Watson \& Andrews, 2002); (2) modelos não adaptacionistas, para os quais a depressão não teria sido selecionada, mas seria um subproduto ou disfunção de outras características adaptativas (Nesse, 2000; Nettle, 2004). Destaca-se que essas abordagens são consideradas evolucionistas, ainda que não adaptacionistas, por enfatizarem a importância da compreensão evolutiva dos sistemas de afetos e do humor deprimido, os quais são a base da depressão. Dessa forma, nossos principais objetivos foram: (1) apresentar teorias evolucionistas da depressão; (2) discutir as relações de complementariedade e contraposição entre essas abordagens; e (3) refletir sobre as possíveis implicações sociais e práticas para o tratamento da depressão. Ao fazê-lo, pretendemos contribuir para a ampliação do debate sobre a depressão a partir de uma perspectiva não patológica, podendo fornecer subsídios para repensar a psicoterapia com o deprimido.

\section{Abordagens evolucionistas da depressão}

\section{Abordagens adaptacionistas}

Uma hipótese adaptacionista propõe que um traço tenha sido selecionado por sua função adaptativa, proporcionando maior aptidão aos indivíduos que o possuíam em relação àqueles que não o possuíam (Andrews, Gangestad, \& Mathew, 2002). Funções adaptativas foram atribuídas a sintomas depressivos, trabalhadas em variados modelos hipotéticos, dos quais destacamos os estudos de Price et al., (1994), Hagen (1999, 2002, 2003), Watson e Andrews (2002) e Andrews e Thomson (2009).

\section{Teoria da competição social}

Partindo de uma visão etológica da espécie humana, Price et al. (1994) defendem a teoria da competição social. Postula-se nesta abordagem que o estado depressivo evoluiu em relação à competição social como uma estratégia perdedora involuntária e inconsciente, permitindo ao indivíduo aceitar a derrota. Isto é, a capacidade de apresentar estados depressivos teria evoluído como um mecanismo para inibir o conflito (Price \& Gardner, 1995). Ao longo do processo evolutivo, aqueles que tinham a capacidade de se tornar depressivos, apresentaram maiores chances de sobrevivência em relação àqueles que não tinham essa capacidade, pois continuar insistindo no conflito poderia levar à morte ou trazer sérios prejuízos. Nesse sentido, o gatilho para a depressão seria a percepção da impossibilidade de vencer o conflito (Price \& Gardner, 1995).

Nessa perspectiva, a característica-chave funcional da depressão é a perda de interesse ou a redução do valor atribuído às atividades anteriormente consideradas significativas. Tal redução favoreceria a diminuição do conflito na medida em que diminuiria o valor atribuído ao recurso pelo qual se compete. Em outras palavras, quanto menor for o valor do recurso para um competidor, maior será a possibilidade de que ele desista da competição (Price et al., 1994). Tal redução ocorreria em função da percepção de diminuição do potencial de conservação de recursos (resource holding potential - RHP), que se refere a uma medida de autoconfiança ou autoestima. $\mathrm{O}$ valor do recurso é determinado pela atratividade daquilo que está sendo disputado. O RHP determina se um indivíduo entra em um confronto ou adota uma estratégia de subordinação involuntária. A diminuição do potencial de retenção de recursos seria percebida como redução da autoestima, levando à forma aguda da depressão, enquanto a manutenção do baixo potencial de conservação de recursos levaria à forma crônica (Price et al., 1994; Price \& Gardner, 1995).

Estudos com roedores fornecem suporte parcial a esse modelo. Apesar de alguns resultados parecerem indicar que um status social específico (dominância ou subordinação) seria o preditor adequado de vulnerabilidade para desenvolver depressão, Larrieu e Sandi (2018) concluíram que a depressão parece ser induzida pela perda de posição social e de recursos. Ou seja, a questão parece residir na perda de um recurso-chave e não apenas na subordinação. No entanto, os autores não descartam a hipótese de que a retirada de recursos por longo prazo possa levar indivíduos subordinados e dominantes à depressão crônica (Larrieu \& Sandi, 2018). 


\section{Hipótese da barganha social}

Hagen $(1999,2002,2003)$ propôs a hipótese da barganha social como modelo explicativo da depressão. De acordo com esse modelo, a depressão teria sido selecionada como resposta às circunstâncias sociais que teriam ocorrido repetidamente no AAE. Para Hagen (2003), circunstâncias sociais predominantemente perigosas ocasionaram pressão seletiva para a evolução de uma estratégia de barganha em humanos. A depressão clínica pode ser uma dessas estratégias com a função de barganha para obrigar que outros membros do grupo forneçam assistência ao deprimido. Dessa forma, na visão de Hagen (2003), “a depressão não deve ser causada simplesmente por perdas, falhas e outros custos sociais, mas também por circunstâncias nas quais os indivíduos não poderiam aliviar unilateralmente esses custos" (p. 105).

Partindo da depressão pós-parto como modelo, Hagen (1999) afirma que as mães que detectam a falta de apoio paterno ou social podem induzir maior investimento do parceiro por meio da depressão. A hipótese foi testada (Hagen, 2002) com 240 mães e pais com um novo filho, utilizando instrumentos de autorrelato. Os resultados evidenciaram que os níveis de depressão pós-parto em um dos cônjuges correlacionaram-se significativamente com maior investimento em educação dos filhos, relatado pelo outro cônjuge. Da mesma forma, uma pesquisa longitudinal realizada por Silva (2008) demonstrou associação entre percepção de baixo suporte social pelas mães, especialmente por parte do pai do bebê, e intensidade elevada de sintomas depressivos no puerpério e aumento do apoio social do parceiro.

\section{Hipótese da navegação social}

Watson e Andrews (2002) propuseram a hipótese da navegação social" (social navigation hypothesis $\mathrm{SNH}$ ) como modelo explicativo da depressão. Segundo a $\mathrm{SNH}$, a depressão tem duas principais funções: resolver problemas sociais complexos e angariar apoio social de parceiros próximos.

A anedonia (redução do interesse pelo ambiente e prazer nas atividades em geral) é um dos critérios obrigatórios no diagnóstico da depressão, estabelecido pelo Manual diagnóstico e estatístico de transtornos mentais: DSM-5 (APA, 2014). Do ponto de vista adaptativo, na perspectiva de Watson e Andrews (2002), a anedonia pode ser compreendida como uma estratégia adaptativa que leva o depressivo a desinvestir energia no ambiente para poupá-la. A energia seria redirecionada para os problemas relacionados à depressão, por duas vias: da ruminação e de apoio social. A ruminação "é um sintoma de depressão que se refere a pensamentos intensos e resistentes à distração" (Bartoskova et al., 2018, p. 1).

Watson e Andrews (2002) usam dois fortes argumentos para sustentar que a depressão seja adaptativa: a alta prevalência da depressão e o aspecto transcultural da depressão (Nettle, 2004). Porém, estudos de cunho epidemiológico têm demonstrado alta correlação entre depressão e doenças infecciosas em indivíduos com doenças (Davydow, Ribe, Pedersen, Vestergaard, \& Fenger-Grøn, 2016; Ivbijaro, Enum, Khan, Lam, \& Gabzdyl, 2014), proporcionando um argumento contrário à hipótese da depressão como traço adaptativo. Ivbijaro et al. (2014) constataram que pessoas com asma, hipertensão, diabetes e coronariopatias sofrem mais internações e geram mais custos ao sistema de saúde quando portadoras de depressão. Davydow et al. (2016) constataram correlação entre depressão unipolar e morte em trinta dias após internação por infecção. Os achados dos autores evidenciam o impacto negativo da depressão nas doenças crônicas. Na população em geral os transtornos depressivos são classificados como maior causa para perda de saúde não fatal (7,5\%) (WHO, 2017).

A partir da $\mathrm{SNH}$, compreende-se a depressão como um mecanismo de defesa, selecionado ao longo da evolução humana e, portanto, relacionado ao contexto no qual apresentou função adaptativa. Dessa forma, a depressão seria evocada pelo contexto e resolvida, caso cumprisse sua função adaptativa (Nettle, 2004; Watson \& Andrews, 2002). Porém a depressão crônica e recorrente depõe contra essa suposta função. As taxas de recorrência podem atingir $50 \%$ entre 3 e 4 anos e $85 \%$ na década seguinte ao episódio depressivo (Baldessarini, 2013). O risco de recaída é muito maior se o tratamento for descontinuado logo após seu início (Baldessarini, Lau, Sim, Sum, \& Sim, 2015). Por outro lado, é preciso considerar que esses estudos se referem ao tratamento farmacológico.

A hipótese de que a depressão é uma adaptação com função de solução de problemas complexos e obtenção de apoio social poderia ser testada a partir da investigação do conteúdo dos pensamentos ruminativos. Se a depressão for, de fato, uma adaptação, esperaríamos que tais estudos identificassem relação entre o conteúdo dos pensamentos ruminativos e os problemas sociais específicos que originaram a depressão.

\section{Hipótese da ruminação analítica}

Andrews e Thomson (2009) propuseram a hipótese da ruminação analítica (analytical rumination hypothesis - ARH), que compreende a depressão como um mecanismo de resposta ao estresse, eliciada por problemas analiticamente difíceis cuja função de resolução ocorre por meio da ruminação.

A partir da ARH, propõe-se que a ruminação ocorra primeiro sobre as causas dos problemas (causal analysis), o que por sua vez promoveria a ruminação para a solução de problemas (problem-solving analysis). A resolução do problema levaria à diminuição dos sintomas da depressão (Bartoskova et al., 2018). Os sintomas da depressão forçam os indivíduos a reduzirem a busca de outras metas e realocarem energia e atenção para analisar 
e resolver o problema desencadeador da depressão (Durisko, Mulsant, \& Andrews, 2015).

Uma limitação dessa hipótese está na definição do que seria um problema analiticamente difícil. Há ainda ausência de evidências de que a ruminação que ocorre durante a depressão seja voltada para a busca de soluções para os problemas. Também não há evidências de que trade-offs seriam realmente necessários para a solução dos problemas, visto que geram altos custos ao deprimido. Ademais, a ARH é limitada, pois nem todos os deprimidos apresentam ruminação (Bartoskova et al., 2018), apesar desta estar inclusa entre os critérios de diagnóstico para um episódio depressivo maior no DSM-V.

Barbic, Durisko e Andrews (2014) desenvolveram uma escala para testar a ARH, denominada Analytical Rumination Questionnaire (ARQ). A escala foi validada por Bartoskova et al. (2018) em dois contextos culturais diferentes (Canadá e República Tcheca). Os resultados confirmaram correlação entre ruminação e a análise causal e de resolução de problemas durante a depressão, dando suporte à ARH como função da depressão.

\section{Abordagens não adaptativas da depressão}

\section{Depressão como desregulação}

Uma revisão de Nesse (2000) discutiu a depressão como consequência mal adaptativa derivada da desregulação dos mecanismos de afetos selecionados tristeza e humor deprimido. Assim, os mecanismos subjacentes à depressão são considerados adaptativos na abordagem da desregulação, no entanto a condição depressão não o é (Kennair, 2018). Nesse (2000) afirma que os organismos dispõem da capacidade de regulação do investimento de esforços e que a tristeza e o humor deprimido poderiam fazer parte desse mecanismo. Tal mecanismo seria ativado diante de situações desfavoráveis, nas quais o esforço para atingir uma meta resultaria em desperdício, perda ou perigo para o próprio indivíduo. Nessas situações, o pessimismo e a falta de motivação derivados do humor deprimido e da tristeza poderiam inibir a ação, evitando o desperdício de energia e as consequências negativas do esforço. Gilbert (2006) aponta que a revisão de Nesse (2000) apresenta a existência de interações complexas entre a busca de objetivos e incentivos, o valor atribuído, a possibilidade de se obter sucesso e as consequências do fracasso.

Apesar de Nesse considerar que as vantagens adaptativas do humor deprimido estão relacionadas à inibição de certos comportamentos sob condições desfavoráveis, para o autor a depressão não levaria às mesmas consequências. A depressão apresenta um pessimismo mais difundido, baixa autoestima e iniciativa reduzida (Nesse, 2000). Essa hipótese de Nesse (2000) foi discutida anos depois em um trabalho de Keller e Nesse (2006). Os autores apresentam a hipótese da congruência situação-sintoma (situation-symptom congruence hypothesis) para explicar por que o humor deprimido teria sido selecionado como resposta a determinados desafios adaptativos. Essa hipótese explica que diferentes sintomas depressivos aparecem diante de situações diferentes para lidar com os desafios específicos de cada situação (Keller \& Nesse, 2006). Por exemplo, a dor ou a tristeza emocional podem aparecer como resposta a perdas de recursos importantes para a aptidão (Nesse, 2000). Para testar essa hipótese foi desenvolvida uma escala denominada Depressive Symptoms Scale (DSS). Os resultados evidenciaram que culpa, ruminação, fadiga e pessimismo eram resultantes de esforços fracassados, enquanto choro, tristeza e necessidade de apoio social ocorriam após perdas sociais (Keller \& Nesse, 2006). Os autores concluem que esses resultados reforçam a hipótese da congruência situação-sintoma, indicando que essas respostas podem ser adaptativas. Admitem, inclusive, que "dependendo da situação, alguns ou mesmo vários episódios de depressão podem ser reações normais a situações fortemente adversas" (Keller \& Nesse, 2006, p. 328). No entanto, os autores reafirmam a necessidade de distinguir os sintomas depressivos adaptativos dos patológicos e realçam a ênfase da abordagem evolucionista no tratamento da causa da depressão em contraposição ao tratamento dos sintomas depressivos, enfatizado em tratamentos tradicionais.

\section{Abordagem das diferenças individuais}

A abordagem das diferenças individuais, proposta por Nettle (2004), apresenta a depressão como não adaptativa e tenta responder por que alguns indivíduos se deprimem enquanto outros não. Em concordância com Nesse (2000), nesse modelo os afetos são considerados adaptações comuns à espécie humana, enquanto a depressão é mal adaptativa. Pressupõe-se, a partir da abordagem evolucionista, que a função adaptativa do humor seja integrar informações sobre o estado recente do ambiente e a condição física atual do organismo, com fins de ajustar suas decisões sobre a alocação do esforço comportamental (Nettle \& Bateson, 2012).

Para explicar o porquê de algumas pessoas se deprimirem e outras não, Nettle (2004) afirma que a evolução produziu uma distribuição populacional contínua de reatividade afetiva. Entende-se por reatividade afetiva a sensibilidade aos estímulos internos e externos aos indivíduos que modulam os estados de humor. As diferenças entre os indivíduos na reatividade afetiva teriam sido selecionadas, dada a diversidade do AAE. Essa variação teria sido adaptativa no AAE, possibilitando aos indivíduos perceberem e responderem de forma mais ajustada ao ambiente social humano, caracteristicamente complexo e dinâmico. Dessa forma, os indivíduos vulneráveis à depressão estariam no limite superior da distribuição populacional.

Uma crítica que pode ser levantada a essa hipótese é que ela é tautológica: algumas pessoas se deprimem 
porque há diferenças individuais, portanto, sabe-se que há diferenças individuais porque algumas pessoas se deprimem e outras não. Isto é, atribui-se a causa da depressão às diferenças individuais e as diferenças individuais à sensibilidade à depressão.

\section{Convergências e controvérsias entre as teorias evolucionistas da depressão}

As hipóteses evolucionistas sobre a depressão trabalhadas nesta revisão trazem leituras variadas da sintomatologia, causas e função da depressão. Contudo, há consenso sobre as emoções serem selecionadas e quanto à relevância do componente social para a depressão. No Quadro 1 apresentamos de forma resumida as principais características das teorias aqui revisadas.

A hipótese da barganha social e a da navegação social atribuem função semelhante à depressão: angariar apoio social dos membros dos grupos. No entanto, a segunda hipótese vai além e trabalha com o aspecto resolutivo da depressão relacionada aos problemas sociais. Este último aspecto é comum nas propostas da hipótese da navegação social e da hipótese da ruminação. Ambas apresentam um dos autores em comum e a segunda parece ser originada a partir da primeira, já que é posterior a ela. Mas divergem em um aspecto-base: na hipótese da navegação social, a função da depressão é dupla: angariar ajuda e resolver problemas socialmente complexos; na ruminação analítica a função da depressão é única: resolver problemas socialmente complexos a partir da análise causal e da análise de soluções para o problema. Muito embora os autores não descartem a possibilidade de que para chegar à solução desses problemas o deprimido possa precisar de apoio social.

Price et al. (1994) demonstraram que a depressão costuma seguir-se a eventos de vida, tais como o luto. Isso aconteceria porque a classificação social é tão dependente do apoio de outros que a perda de pessoas significativas se torna o principal preditor de perda de classificação no ranking social. O provimento de ajuda por parte dos parceiros tem sido testado no caso da depressão pós-parto e há indícios, resultantes de pesquisas empíricas, de que os parceiros de mães com depressão pós-parto "compensem" a ausência materna "investindo" mais nos bebês (Bottino, Nadanovsky, Moraes, Reichenheim, \& Lobato, 2012). Mas faltam evidências científicas de que a depressão que ocorre em outras situações pode levar a angariar apoio social. Faltam evidências também de que a depressão induza mudanças cognitivas que levam à análise e à solução de problemas sociais. O desenvolvimento da escala de ruminação analítica (ARQ) é um passo que contribui para responder a essa questão, no entanto apresenta limitações por ser um estudo ainda recente e sem evidências de aplicação prática e em outras populações (Bartoskova et al., 2018).

Quadro 1. Abordagens evolucionistas da depressão

\begin{tabular}{|c|c|c|c|c|c|}
\hline Tipo/modelo & Hipótese & $\begin{array}{c}\text { Causas/eliciadores } \\
\text { da depressão }\end{array}$ & Função da depressão & Definição & $\begin{array}{c}\text { Autores } \\
\text { (principais) }\end{array}$ \\
\hline \multirow{4}{*}{ 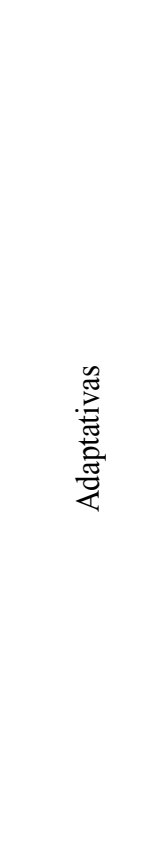 } & $\begin{array}{l}\text { Hipótese da } \\
\text { competição } \\
\text { social }\end{array}$ & $\begin{array}{l}\text { Na forma crônica aparece em } \\
\text { função do baixo "potencial } \\
\text { de conservação de recursos", } \\
\text { e na forma aguda, em função } \\
\text { da queda do "potencial de } \\
\text { conservação de recursos" }\end{array}$ & $\begin{array}{l}\text { Resolução de conflitos } \\
\text { que envolvem } \\
\text { competição social }\end{array}$ & $\begin{array}{l}\text { Estratégia involuntária } \\
\text { de subordinação. }\end{array}$ & $\begin{array}{l}\text { Price et al. } \\
(1994)\end{array}$ \\
\hline & $\begin{array}{l}\text { Hipótese da } \\
\text { barganha } \\
\text { social }\end{array}$ & $\begin{array}{l}\text { É acionada quando os } \\
\text { indivíduos percebem que } \\
\text { estão sofrendo custos que } \\
\text { podem ser aliviados pelas } \\
\text { ações dos membros do grupo }\end{array}$ & $\begin{array}{l}\text { Sinaliza aos outros } \\
\text { membros do grupo } \\
\text { que alguém está } \\
\text { sofrendo custos }\end{array}$ & $\begin{array}{l}\text { É uma estratégia } \\
\text { (inconsciente) de } \\
\text { barganha (negociação) } \\
\text { em humanos }\end{array}$ & $\begin{array}{l}\text { Hagen (1999, } \\
2002,2003)\end{array}$ \\
\hline & $\begin{array}{l}\text { Hipótese da } \\
\text { navegação } \\
\text { social }\end{array}$ & Conflito social & $\begin{array}{l}\text { Angariar apoio social } \\
\text { e resolver problemas } \\
\text { sociais complexos }\end{array}$ & $\begin{array}{l}\text { É um tipo de dor } \\
\text { emocional }\end{array}$ & $\begin{array}{l}\text { Watson e } \\
\text { Andrews } \\
(2002)\end{array}$ \\
\hline & $\begin{array}{l}\text { Hipótese da } \\
\text { ruminação } \\
\text { analítica }\end{array}$ & $\begin{array}{l}\text { Eliciada por problemas } \\
\text { analiticamente difíceis }\end{array}$ & $\begin{array}{l}\text { Resolução de problemas } \\
\text { analiticamente difíceis } \\
\text { por meio da análise } \\
\text { causal e solução } \\
\text { de problemas }\end{array}$ & $\begin{array}{l}\text { Um mecanismo de } \\
\text { resposta ao estresse }\end{array}$ & $\begin{array}{l}\text { Andrews e } \\
\text { Thomson } \\
(2009)\end{array}$ \\
\hline
\end{tabular}


Quadro 1. Continuação

\begin{tabular}{|c|c|c|c|c|c|}
\hline Tipo/modelo & Hipótese & $\begin{array}{c}\text { Causas/eliciadores } \\
\text { da depressão }\end{array}$ & Função da depressão & Definição & $\begin{array}{c}\text { Autores } \\
\text { (principais) }\end{array}$ \\
\hline \multirow[t]{2}{*}{ 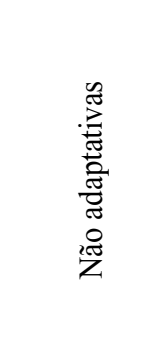 } & $\begin{array}{l}\text { Diferenças } \\
\text { individuais }\end{array}$ & $\begin{array}{l}\text { Existe uma distribuição de } \\
\text { reatividade ideal dos sistemas } \\
\text { de afetos na população. } \\
\text { Estar no limite superior } \\
\text { dessa distribuição leva } \\
\text { à vulnerabilidade }\end{array}$ & - & $\begin{array}{l}\text { É uma desregulação } \\
\text { que aparece em } \\
\text { indivíduos vulneráveis }\end{array}$ & Nettle (2004) \\
\hline & Desregulação & Eventos graves de vida & - & $\begin{array}{l}\text { É uma desregulação } \\
\text { dos afetos. }\end{array}$ & Nesse (2000) \\
\hline
\end{tabular}

\section{A depressão é adaptativa?}

As discussões acerca das abordagens evolucionistas da depressão pesam, sobretudo, quanto à possibilidade de apresentar função adaptativa, tendo em vista seu alto custo para os indivíduos. As adaptações, segundo Nettle (2004), caracterizam-se por terem: (1) surgido em uma população ancestral; (2) melhorado a aptidão daqueles indivíduos que a apresentaram; e (3) assim se espalhado para alcançar a fixação genética. Porém, para o autor, a depressão não mostra nenhuma dessas marcas, pois é caracterizada pela hereditariedade, recorrência, comprometimento cognitivo e impacto negativo sobre as relações sociais. Entretanto, em contraposição a essa crítica, apontamos que nem todas as características adaptativas são positivas ou geram apenas efeitos positivos no ambiente moderno. Werner (2018) aponta que "não devemos confundir adaptação biológica com felicidade ou com saúde mental ou física" (p. 19). A dor física e a náusea são exemplos de adaptações projetadas para proteger de danos, apesar de não serem experiências agradáveis (Hagen, 2011). Outro exemplo é a seleção para preferência pelo sabor doce, que pode ter sido adaptativa ao fornecer uma "pista de que o alimento contém carboidrato e energia" (Lopes, Ferreira, \& Araújo, 2018, p. 533). Mas no ambiente moderno, com a disposição abundante de açúcares, a preferência pelo sabor doce pode contribuir para o aumento de comorbidades como diabetes, obesidade e outras doenças crônicas, que geram sérios prejuízos sociais e elevados gastos em saúde pública.

Um argumento favorável à hipótese da depressão como característica adaptativa é apresentado por Hagen (2011). O autor argumenta que as adaptações são universais para uma espécie, isto é, devem estar presentes no cotidiano e não apenas em populações clínicas. A depressão é um problema de saúde pública que atinge milhões de pessoas ao redor do mundo e, mesmo em culturas mais tradicionais, tem havido relatos de sua ocorrência (Watson \& Andrews, 2002). Quanto à ausência de variabilidade genética, requerida para considerar-se um traço como adaptativo, Hagen (2003) afirma que os estudos sobre depressão devem ser conciliados com os correlatos em genética e bioquímica. Estudos com genoma humano têm evidenciado que existem dificuldades em determinar os genes associados ao transtorno de depressão maior (TDM) devido à heterogeneidade etiológica característica da depressão (Sullivan, 2015). Um estudo elaborado por Ripke et al. (2013) com genoma e TDM analisou mulheres com TDM recorrente. Os resultados levaram à hipótese de que as interações sociais e genes são particularmente importantes para TDM e que este só pode ser entendido se fatores de risco genéticos e ambientais forem modelados simultaneamente (Ripke et al., 2013). Em outro estudo realizado por Howard et al. (2018) os resultados mostraram que o fenótipo da depressão analisado tinha um componente genético significativo, indicando herdabilidade. Os resultados desses estudos indicaram uma provável variabilidade genética na depressão, sendo desfavorável à hipótese adaptativa da depressão.

A abordagem da depressão enquanto desregulação afirma que a tristeza e o humor deprimido são adaptativos (Nesse, 2000), porém a depressão é uma desregulação. Se assim for, é preciso estabelecer maior compreensão sobre as funções adaptativas da tristeza e do humor deprimido para a saúde a fim de compreender-se como estes se tornam disfuncionais (Hagen, 2011). De toda sorte, a teoria da desregulação tem implicações clínicas para o tratamento do deprimido. A partir da teoria da desregulação, o objetivo principal da psicoterapia seria a restauração da tristeza e do humor deprimido ao "limiar" considerado saudável em vez da redução dos sintomas da depressão. Mas qual seria esse limite? É possível argumentar que esse "limite" faça referência à funcionalidade e ao bem-estar do paciente. No entanto, certamente, essa não é uma questão de fácil resolução.

\section{Implicações sociais e práticas}

As teorias evolucionistas da depressão, se confirmadas, como alguns estudos empíricos citados ao longo desse texto vêm demonstrando, oferecem fortes implicações para os estudos de saúde mental. A depressão, até hoje, tem sido amplamente aceita e 
descrita pelo DSM-5 (APA, 2014) como uma desordem mental. Porém, se a hipótese da ruminação analítica, por exemplo, estiver correta, abre-se a possibilidade para novos estudos a partir de uma perspectiva não patológica da depressão. Algumas terapias na psicologia trabalham com a modificação dos pensamentos ruminativos de deprimidos. No entanto, partem do princípio de que a ruminação é um aspecto negativo que deve ser suprimido. Em contraposição, a hipótese da navegação social e a hipótese da ruminação analítica pressupõem que a ruminação seja um fator primordial na depressão, devendo ser explorado e não suprimido. Um teste da teoria da ruminação analítica poderia envolver a comparação entre dois grupos de deprimidos, recebendo um deles tratamento baseado na modificação dos pensamentos ruminativos e 0 outro enfocando a análise causal e resolução de problemas revelados a partir dos pensamentos ruminativos.

No tocante ao trabalho psicoterapêutico de psicólogos evolucionistas com deprimidos, destaca-se os estudos de Giosan (2020), que recentemente publicou um manual sobre a evolutionary cognitive therapy (CET) para depressão (em uma tradução livre: terapia cognitiva evolucionista). A CET é resultado da junção de algumas técnicas da terapia cognitivo-comportamental (TCC) com a psicologia evolucionista como base da compreensão da depressão. A CET foi testada clinicamente em um estudo randomizado em comparação com a terapia cognitiva (Giosan et al., 2020). Os resultados mostraram semelhanças entre a efetividade em ambas as terapias, no entanto, a CET foi estatisticamente superior à TCC, provocando maior envolvimento em atividades sociais e agradáveis, que se mantiveram no follow-up, e maior redução na inibição comportamental/evasão (Giosan et al., 2020). A CET tem por foco identificar problemas relacionados à aptidão (fitness) e planejar intervenções apropriadas para esses problemas, com o objetivo de ajudar os indivíduos a atingirem seus objetivos biossociais (Giosan, 2020). Considera-se aqui o termo aptidão no sentido evolutivo, como o grau de conformidade entre um organismo e o meio ambiente (Giosan, Mureșan, Wyka, Cobeanu, \& Szentagotai, 2018).

Outro exemplo é o estudo de caso publicado por Krupnik (2015) sobre os resultados do uso da dessensibilização e reprocessamento do movimento ocular (EMDR) com uma terapia de base evolucionista para a depressão, denominada tratamento da depressão em declive (TDD). A TDD trabalha em três fases: exploração, aceitação e ativação comportamental. As duas primeiras fases consistem em facilitar a resposta depressiva por meio da experiência de aceitação e, no terceiro momento, a motivação é trabalhada por meio da experiência do prazer, incentivando o envolvimento em atividades prazerosas. Krupnik realizou dezesseis sessões de terapia com um paciente com histórico prévio de depressão e outros transtornos. Ao final da intervenção, o paciente apresentou melhoras que se mantiveram estáveis no follow-up.
A perspectiva evolucionista também aponta para as mudanças que podem ser feitas no ambiente, de forma a diminuir o sofrimento e a vulnerabilidade à depressão (Nettle \& Bateson, 2012). Como citado no início desta revisão, o risco de tornar-se deprimido é aumentado pela pobreza, desemprego, eventos de vida, doença física e problemas causados por uso de álcool e drogas (WHO, 2017). Trabalhar questões humanitárias, diminuição da desigualdade social, políticas mais igualitárias e alocação de mais recursos nas políticas de álcool e drogas pode ter efeito protetivo para as pessoas que estão em maior risco.

É importante mencionar que as aplicações da psicologia evolucionista em expansão evidenciam a superação do modelo de "duas culturas" para o modelo biossocial, argumentando contra o pensamento dicotômico que enfatiza os aspectos biológicos ou o ambiente social. Por exemplo, Shakespeare e Erickson (2001) criticam modelos ultradarwinistas que levam consigo a legitimidade das ciências naturais ao defender a predominância do imperativo biológico, bem como o outro extremo, com modelos limitados ao construcionismo social. Teóricos evolucionistas atuais propõem uma abordagem interdisciplinar que inclui estudos do cérebro deprimido com pesquisas psicológicas sobre seus correlatos ecológicos, desenvolvimentais e biocomportamentais para explicar o humor deprimido e suas manifestações clínicas (Badcock, Davey, Whittle, Allen, \& Friston, 2017). Nesta síntese teórica de sistemas dinâmicos a depressão só pode ser entendida considerando os mecanismos neurais em interação bidirecional com o contexto mais amplo da evolução humana em processos de enculturação, desenvolvimento, corporeamento e comportamento. Destaca-se que a explicação da depressão a partir do funcionamento biológico integrado ao ambiente não invalida as demais áreas da saúde mental, que têm, ao longo de décadas, avançado em tratamentos que beneficiam a qualidade de vida das pessoas com depressão. Contudo, uma visão sinérgica sobre a depressão pode informar como melhor lidar com essa condição ao passo que busca capturar interações complexas em múltiplos níveis causais proximais e distais.

\section{Considerações finais}

A depressão não é uma doença única, pois existem diferentes tipos de transtornos depressivos - transtorno disruptivo da desregulação do humor, transtorno depressivo maior, transtorno depressivo persistente (distimia), transtorno disfórico pré-menstrual, transtorno depressivo induzido por substância/medicamento, transtorno depressivo devido a outra condição médica, outro transtorno depressivo especificado e transtorno depressivo não especificado (APA, 2014) e, por conseguinte, diferentes situações que evocam um quadro depressivo. A psicologia evolucionista possui uma literatura considerável sobre os mecanismos evoluídos da depressão, os quais fornecem 
indícios para se repensar a perspectiva psicopatológica da depressão. As teorias evolucionistas adaptativas, em especial, oferecem um referencial teórico que busca explicar a depressão a partir de sua função adaptativa e, portanto, não necessariamente como um traço patológico.

Trabalhos psicoterapêuticos com deprimidos a partir de uma perspectiva evolucionista têm sido realizados (e.g., Giosan, 2020; Giosan et al., 2020; Krupnik, 2015) e escalas que testam as previsões das hipóteses evolucionistas têm sido desenvolvidas, a exemplo daquelas citadas ao longo desta revisão (e.g., Barbic, Durisko, \& Andrews, 2014; Bartoskova et al., 2018; Keller \& Nesse, 2006). No entanto, ainda não existem relatos no Brasil da validação dessas escalas e nem da implementação de trabalhos psicoterapêuticos nessa abordagem com a depressão. Espera-se que esta revisão possa fomentar essa discussão e pesquisa.

Esta revisão apresenta limitações, já que não foram discutidas todas as hipóteses evolucionistas sobre a depressão e não houve um levantamento exaustivo de evidências científicas delas. De qualquer forma, não foi nosso objetivo responder definitivamente as questões que envolvem as teorias evolucionistas sobre a depressão ou propor uma nova teoria. Ao contrário, sugerimos que mais estudos empíricos sejam realizados no sentido de submeter as teorias evolucionistas a teste, considerando as implicações e os benefícios que podem resultar delas. Assim, reafirmamos a relevância dos estudos teóricos da depressão sob abordagens evolucionistas e a eminente necessidade de estudos empíricos que as submetam a testagem.

\section{Evolutionary theories of depression: overview and perspectives}

Abstract: Depression has reached epidemic levels worldwide. Would that be a mental disorder, as claimed by consensus on mental health? Evolutionary theorists have questioned the function of depression and proposed specific models to explain it. The aim of this paper is to present the evolutionary theories of depression, to discuss the complementarity and contradictions between these theories, and to present the social and practical implications for the treatment of depression. Those reflections and issues in the field of mental health may influence further studies from a non-pathological perspective of depression. In the field of psychology, this perspective provides insights to reevaluate psychotherapy to treat depression by focusing on causal analysis and problem solving. The study suggests that new empirical studies should be conducted to test and systematize evolutionary theories of depression.

Keywords: evolutionary psychology, depression, adaptation, deregulation, mental health.

\section{Théories évolutionnistes de la dépression : aperçu et perspectives}

Résumé : La dépression a atteint les proportions d'une épidémie mondiale. Les théoriciens évolutionnistes se sont interrogés sur la fonction de la dépression et ont proposé des modèles spécifiques pour l'expliquer. Cet article vise à présenter les différentes théories évolutionnistes de la dépression, discuter leurs relations de complémentarité et d'opposition, et débattre les possibles implications sociales et pratiques pour le traitement de la dépression. Ces réflexions dans le domaine de la santé mentale peuvent influencer de nouvelles études dans une perspective non pathologique de la dépression. Dans le domaine de la psychologie, cette perspective permet de repenser la psychothérapie avec les personnes déprimées, en se concentrant sur l'analyse causale et la résolution des problèmes. Des études empiriques sont proposées dans le but de tester et systématiser les théories évolutionnistes de la dépression.

Mots-clés : psychologie évolutionniste, dépression, adaptation, déréglementation, santé mentale.

\section{Teorías evolutivas de la depresión: visión general y perspectivas}

Resumen: La depresión ha alcanzado niveles epidemiológicos alrededor del mundo. ¿Será la depresión un trastorno mental como se cree en el dominio de la salud mental? Teóricos evolucionistas se cuestionan sobre la depresión y han propuesto modelos específicos para explicarla. El objetivo de este artículo fue presentar teorías evolutivas de la depresión, discutir las relaciones de complementariedad y contraposición entre ellas, así como las implicaciones sociales y prácticas para su tratamiento. Estas reflexiones y cuestionamientos, en el dominio de la salud mental, pueden influir en nuevos estudios a partir de una perspectiva no patológica de la depresión. En el ámbito de la psicología, esta perspectiva provee subsidios para repensar la psicoterapia con el deprimido enfocándose en el análisis causal y la resolución de problemas. Se sugiere la realización de estudios empíricos con la finalidad de probar y sistematizar las teorías evolucionistas de la depresión.

Palabras clave: psicología evolutiva, depresión, adaptación, desregulación, salud mental. 


\section{Referências}

Abed, R., Ayton, A., John-Smith, P., Swanepoel, A., \& Tracy, D. K. (2019). Evolutionary biology: An essential basic science for the training of the next generation of psychiatrists. British Journal of Psychiatry, 215(6), 699-701. doi: 10.1192/bjp.2019.123

American Psychiatric Association. (2014). Manual diagnóstico e estatístico de transtornos mentais: DSM-5 (5a ed.). Porto Alegre, RS: Artmed.

Andrews, P. W., Gangestad, S. W., \& Mathew, D. (2002). Adaptationism: How to carry out an exaptationist program. Behavioral and Brain Sciences, 25(4), 489-504. doi: 10.1017/S0140525X02000092

Andrews, P. W., \& Thomson, J. A., Jr. (2009). The bright side of being blue: Depression as an adaptation for analyzing complex problems. Psychological Review. doi: $10.1037 / \mathrm{a} 0016242$

Badcock, P. B., Davey, C. G., Whittle, S., Allen, N. B., \& Friston, K. J.(2017). The depressed brain: An evolutionary systems theory. Trends in Cognitive Sciences, 21(3), 182-194. doi: 10.1016/j.tics.2017.01.005

Baldessarini, R. J. (2013). Chemotherapy in psychiatry: pharmacologic basis of treatments for major mental illness. New York: Springer. doi: 10.1007/978-1-4614-3710-9

Baldessarini, R. J., Lau, W. K., Sim, J., Sum, M. Y., \& Sim, K. (2015). Duration of initial antidepressant treatment and subsequent relapse of major depression. Journal of Clinical Psychopharmacology, 35(1), 75-76. doi: 10.1097/JCP.0000000000000263

Barbic, S. P., Durisko, Z., \& Andrews, P. W. (2014). Measuring the bright side of being blue: A new tool for assessing analytical rumination in depression. PLoS ONE, 9(11), 1-9. doi: 10.1371/journal.pone.0112077

Bartoskova, M., Sevcikova, M., Durisko, Z., Maslej, M. M., Barbic, S. P., Preiss, M., \& Andrews, P. W. (2018). The form and function of depressive rumination. Evolution and Human Behavior, 39(3), 277-289. doi: 10.1016/ j.evolhumbehav.2018.01.005

Bottino, M. N., Nadanovsky, P., Moraes, C. L., Reichenheim, M. E., \& Lobato, G. (2012). Reappraising the relationship between maternal age and postpartum depression according to the evolutionary theory: Empirical evidence from a survey in primary health services. Journal of Affective Disorders, 142(1-3), 219-224. doi: 10.1016/ j.jad.2012.04.030

Cosmides, L., Tooby, J., \& Barkow, J. H. (1992). Introduction: Evolutionary psychology and conceptual integration. In J. H. Barkow, L. Cosmides, \& J. Tooby (Eds.), The adapted mind: evolutionary psychology and the generation of culture (pp.3-15). Oxford: Oxford University Press.

Davydow, D. S., Ribe, A. R., Pedersen, H. S., Vestergaard, M., \& Fenger-Grøn, M. (2016). The association of unipolar depression with thirty-day mortality after hospitalization for infection: A population-based cohort study in Denmark. Journal of Psychosomatic Research, 89, 32-38. doi: 10.1016/j.jpsychores.2016.08.006
Durisko, Z., Mulsant, B. H., \& Andrews, P. W. (2015). An adaptationist perspective on the etiology of depression. Journal of Affective Disorders, 172, 315323. doi: 10.1016/j.jad.2014.09.032

Gilbert, P. (2006). Evolution and depression: Issues and implications. Psychological Medicine, 36(3), 287-297. doi: 10.1017/S0033291705006112

Giosan, C. (2020). Cognitive evolutionary therapy for depression: therapy manual. New York: Springer. doi: 10.1007/978-3-030-38874-4_5

Giosan, C., Cobeanu, O., Wyka, K., Muresan, V., Mogoase, C., Szentagotai, A., Malta, L. S., \& Moldovan, R. (2020). Cognitive evolutionary therapy versus standard cognitive therapy for depression: a single-blinded randomized clinical trial. Journal of Clinical Psychology, 76(10), 1818-1831. doi: 10.1002/jclp.22991

Giosan, C, Mureșan, V., Wyka, K., Cobeanu, O., \& Szentagotai, A. (2018). The evolutionary fitness scale: A measure of the independent criterion of fitness. EvoS Journal: The Journal of the Evolutionary Studies Consortium, 8(1), 181-205.

Hagen, E. H. (1999). The functions of postpartum depression. Evolution and Human Behavior, 20(5), 325-359. doi: 10.1016/S1090-5138(99)00016-1

Hagen, E. H. (2002). Depression as bargaining: the case postpartum. Evolution and Human Behavior, 23(5), 323-336. doi: 10.1016/S1090-5138(01)00102-7

Hagen, E. H. (2003). The bargaining model of depression. In P. Hammerstein (Ed.), Genetic and cultural evolution of cooperation (pp. 95-123). Cambridge, MA: MIT Press.

Hagen, E. H. (2011). Evolutionary theories of depression: A critical review. Canadian Journal of Psychiatry, 56(12), 716-726. doi: 10.1177/070674371105601203

Howard, D. M., Adams, M. J., Shirali, M., Clarke, T. K., Marioni, R. E., Davies, G.,... McIntosh, A. M. (2018). Genome-wide association study of depression phenotypes in UK biobank identifies variants in excitatory synaptic pathways. Nature Communications, 9(1), 1-10. doi: 10.1038/s41467-018-03819-3

Ivbijaro, G. O., Enum, Y., Khan, A. A., Lam, S. S. K., \& Gabzdyl, A. (2014). Collaborative care: Models for treatment of patients with complex medical-psychiatric conditions. Current Psychiatry Reports, 16(11) 1-12. doi: 10.1007/s11920-014-0506-4

Izar, P. (2018). Fundamentos da evolução do comportamento. In M. E. Yamamoto \& J. V. Valentova (Eds.), Manual de psicologia evolucionista (pp. 56-74). Natal, RN: EDUFRN.

Keller, M. C., \& Nesse, R. M. (2006). The evolutionary significance of depressive symptoms: different adverse situations lead to different depressive symptom patterns. Journal of Personality and Social Psychology, 91(2), 316-330. doi: 10.1037/0022-3514.91.2.316

Kennair, L. E. O. (2003). Evolutionary psychology and psychopathology. Current Opinion in Psychiatry, 16(6), 691-699. doi: 10.1097/00001504-200311000-00015 
Kennair, L. E. O. (2018). Psicopatologia evolucionista. In M. E. Yamamoto \& J. V.Valentova (Eds.), Manual de psicologia evolucionista (pp. 255-270). Natal, RN: EDUFRN.

Krupnik, V. (2015). Integrating EMDR into an evolutionarybased therapy for depression: A case study. Clinical Case Reports, 3(5), 301-307. doi: 10.1002/ccr3.228

Laland, K. N., \& Brown, G. R. (2002). Sense and nonsense. New York: Oxford University Press.

Larrieu, T., \& Sandi, C. (2018). Stress-induced depression: Is social rank a predictive risk factor? BioEssays, 40(7): 1800012. doi: 10.1002/bies.201800012

Lopes, F. de A., Ferreira, D. Q. C., \& Araújo, A. (2018). Comportamento alimentar. In M. E. Yamamoto \& J. V. Valentova (Eds.), Manual de psicologia evolucionista (pp. 523-547). Natal, RN: EDUFRN.

Nesse, R. M. (2000). Is depression an adaptation? Archives of general psychiatry, 57(1), 14-20. doi: 10.1001/ archpsyc.57.1.14

Nettle, D. (2004). Evolutionary origins of depression: A review and reformulation. Journal of Affective Disorders, 81(2), 91-102. doi: 10.1016/j.jad.2003.08.009

Nettle, D., \& Bateson, M. (2012). The evolutionary origins of mood and its disorders. Current Biology, 22(17), R712-R721. doi: 10.1016/j.cub.2012.06.020

Price, J., \& Gardner, R. (1995). The paradoxical power of the depressed patient: A problem for the ranking theory of depression. British Journal of Medical Psychology, 68(3), 193-206. doi: 10.1111/j.2044-8341.1995.tb01827.x

Price, J., Sloman, L., Gardner, R., Gilbert, P., \& Rohde, P. (1994). The social competition hypothesis of depression. British Journal of Psychiatry, 164(3), 309-315. doi: 10.1192/bjp.164.3.309

Resende, B. D. de. (2011). Contribuições da perspectiva evolucionista para a gerontologia. Revista Kairós, 14(1), 99-107. doi: 10.23925/2176-901X.2011v14i1p99-107

Ripke, S., Wray, N. R., Lewis, C. M., Hamilton, S. P., Weissman, M. M., Breen, G., ... McIntosh, A. (2013). A mega-analysis of genome-wide association studies for major depressive disorder. Molecular Psychiatry, 18(4), 497-511. doi: 10.1038/mp.2012.21
Shakespeare, T., \& Erickson, M. (2001). Different strokes: Beyond biological determinism and social constructionism. In H. Rose \& S. Rose (Eds.), Alas poor Darwin: arguments against evolutionary psychology (pp. 190-205). London: Vintage.

Silva, G. A. da. (2008). Estudo longitudinal sobre prevalência e fatores de risco para depressão pós-parto em mães de baixa renda (Dissertação de Mestrado, Universidade de São Paulo). Recuperado de https://doi. org/10.11606/D.47.2008.tde-29072009-162342

Sloman, L., Gilbert, P., \& Hasey, G. (2003). Evolved mechanisms in depression: the role and interaction of attachment and social rank in depression. Journal of Affective Disorders, 74(2), 107-121. doi: 10.1016/ S0165-0327(02)00116-7

Sullivan, P. F. (2015). Associations with depression. Nature, 523(7562), 539-540. doi: 10.1038/nature14635

Varella, M. A. C., \& Valentova, J. V. (2018). Glossário. In E. Yamamoto \& J. V. Valentova (Eds.), Manual de psicologia evolucionista (pp. 596-573). Natal, RN: EDUFRN.

Watson, P. J., \& Andrews, P. W. (2002). Toward a revised evolutionary adaptationist analysis of depression: the social navigation hypothesis. Journal of Affective Disorders, 72(1), 1-14. doi: 10.1016/S0165-0327(01)00459-1

Werner, D. (2018). Prefácio. In M. E. Yamamoto \& J. V. Valentova (Eds.), Manual de psicologia evolucionista (pp. 7-25). Natal: EDUFRN.

World Health Organization. (2017). Depression and other common mental disorders - Global health estimates. Geneva: Autor.

Yamamoto, M. E. (2018). Histórico e plano do livro. In M. E. Yamamoto \& J. V. Valentova (Eds.), Manual de psicologia evolucionista (pp. 29-54). Natal, RN: EDUFRN. 